\title{
Ant Colony Optimization with the Fusion of Adaptive K- means and Gaussian Second Derivative for Image Segmentation
}

\author{
Pragya Sharma \\ Dept. of CSE/IT \\ MPCT College
}

\author{
Unmukh Datta \\ Dept. of CSE/IT \\ MPCT College
}

\begin{abstract}
In this study, proposed an ant colony optimization (ACO) with the fusion of adaptive k-means and Gaussian second derivative for image segmentation. With the use of two algorithms will enhance the segmentation accuracy and speed up algorithm convergence. In the Gaussian second derivative, it is used for enhancing edges of an image because some information loses in the previous algorithm. The experimental process proved that a new hybrid clustering algorithm is more efficient than previous algorithms. Principally, this algorithm has better results in image segmentation. The proposed method can get profit of the K-means clustering for image segmentation in the aspects of less execution time. Also, it can get the benefits of ACO in the aspects of f-measure accuracy.
\end{abstract}

\section{Keywords}

ACO, Gaussian Second Derivative and K-means.

\section{INTRODUCTION}

Image segmentation task is to group pixels in homogeneous areas by use of the common function technique. Features can be represented through gray levels, color and texture space every exploring similarities between pixels of an area. Segmentation [1] refers to the partitioning a digital image system into more than one area. The intention of segmentation is to simplify and alter an image representation into approximately that is more significant and easier to research. Image segmentation is almost always used to detect objects and boundaries (curves, lines, and many others.) in images. In the state-of-the-art world computer vision has become an interdisciplinary area and its applications will also be observed many like subject be it medical, remote sensing, electronics and so forth. The effect of image segmentation is a set of areas that jointly quilt the whole photo, or a contour collection mined from the image. Every pixel in an area is equivalent with respect to a few attribute or computed property, for example texture, color, or intensity.[2]

\section{IMAGE SEGMENTATION TECHNIQUES}

\subsection{K-Means Algorithm}

This algorithm clusters point nearest to the centroid. The centroid is basically the average of each point in that cluster and has coordinate as the arithmetic mean over all points in the cluster, separately for each dimension.

Although hierarchical methods can be more accurate, partitional methods are used in applications involving large data sets (related to images) due to the fact of construction of a dendrogram is computationally prohibitive. [3]. In hierarchal method if once the merge/split is done, it can never be undone.

\subsection{Ant Colony Optimization}

An ant colony algorithm is a new optimization approach through simulating evolution. The ant clustering algorithm is a branch, which is a clustering algorithm type situated on swarm intelligence and is typically utilized in image segmentation. Researchers found the fact that ants can categorize their ant babies automatically and orderly. Researchers proposed the ant clustering algorithm. Its common concept is that everyone unclustering objects are put on a two-dimensional grid. All object receipts an initial place randomly. Ants are also for grid and also can be one place to another place to move randomly. When an ant runs into an object, it will measure colony similarity within its local range. This information will be transformed into the probability that the ant moves the object by. The ant will drop or pick up the object according to probability value. The objects, transportation through ants is a self organization clustering process. In the end, various clusters on the flat will take shape. [4]

\section{LITERATURE SURVEY}

The enhanced ant colony algorithm was achieved through improving tendency of ant to move in one-of-a-kind path in probabilistic selection rule. This algorithm indicates a balance between the ant's direction and distribution of pheromone. The region detected as the tumor is more distinct, clear and without any additional margin, which is typically brought in via inflammation [5].

Ms. Aparna (2014) et al present that The Data Clustering idea is considered to be most important in the numerous areas of application like Bioinformatics, text mining, fraud detection, health care, image processing.Various algorithms come under partitional clustering among which Bisecting K-Means are an excellent one that provide a good quality output for clustering large number of data.[6].

This method uses an ACO technique alongside K-Means and Level set to locate the liver region. The method was placed on CT data sets and the algorithm performance was measured applying the receiver operating characteristics such as fmeasure and sensitivity. Quantitative analysis suggests that this method can consistently segment liver images with high accuracy and efficiency. [1].

Dharmendra K Roy and Lokesh K Sharma [7] presented algorithm of clustering based on the paradigm of Genetic kmeans that works well for information with categorical features and mixed numeric features.

Shi $\mathrm{Na}$ et al. [8] discussed the standard k-means clustering algorithm and analyzed the shortcomings of the standard kmeans algorithm. 
Singh et.al [9] in this work, a fully automated liver segmentation technique is described which means that this technique identifies the desired Region of Interest itself. The performance of k-means and level set, and proposed hybrid kmeans and level set was evaluated on a number of CT images to segment the liver region. The segmentation requires prior anatomical knowledge of the abdominal CT image to differentiate between different organs of the human body. Both quantitative and qualitative analyses are in favor of hybrid k-means (k-means with ACO). The analyses resulted in high F-measure rate and sensitivity in all testing data. This technique can further be used on other modalities with different diseases, which will be helpful in surgeries. Also colored images can be used and implementation can be done in 3-D. The performance of the proposed algorithm depends on the choice of initial centroids as well as on proper selection of a structural element.

Bhagwati Charan Patel presents an attempt is prepared to clustering algorithm of k-means implement for breast image segmentation for the micro calcification detection and also a decision system of computer based for breast cancer detection in an improved way. Developed decision of computer aided systems for micro calcification detection in mammogram images the system is detecting micro calcifications capable through digital mammograms inspection. The selection of feature is a number based, shape and color of objects present in the image. Bins values number, Class number, object sizes, is considered as appropriate features for image retrieval knowledge. The detection accuracy was estimated and also compared with present works and it has been reported that improved accuracy if the algorithm of $\mathrm{K}$-means is adaptively implemented. The results are found to be suitable when subjected to radiologists for their validation. [10]

ACO metaheuristic is a new population-based method inspired through the observation of real ant colonies and also based upon their foraging behavior collective. In the ACO, problem solutions are constructed within the stochastic iterative procedure, through the components of the adding solution to the partial solutions. All individual ants construct a solution part applying pheromone of artificiality, which reflects its experience accumulated while solving the issue, and also heuristic data dependent. In this paper, we applied the ACO idea for discrete optimization in the MRF-based image segmentation. Our basic motivation is that ACO metaheuristic has been effectively applied to various NP-hard combinatorial optimization issue and has been present to be competitive against conventional optimization approaches like SA and GA. In this paper, we will use the algorithm which uses both ACS with MRF in which an artificial ant searches colony for an optimal labeling of the image pixels that maximizes MAP estimate. Individual ant constructs a candidate partition, through a relaxation labeling with respect to contextual constraints. After some iteration, the partition representing the energy function optimum value is obtained. [11] ragged.

\section{PROPOSED METHODOLOGY}

In the proposed work, first take an input of any image. Then apply Gaussian second derivative for determining horizontal and vertical direction and this will improve in the ACO algorithm at the time of gradient. Assign all points to nearby cluster center based on the minimum distance through checking distance between cluster centers and point, then recompute the novel centers of clusters. It repeats until few convergence criteria is met. K-means Algorithm is minimizing the total distance of data points to the cluster center, of the cluster they are assigned to. Also, it does not require the actual computation of distances. A k-means algorithm disadvantage is that the number of desired clusters needs to be set before.

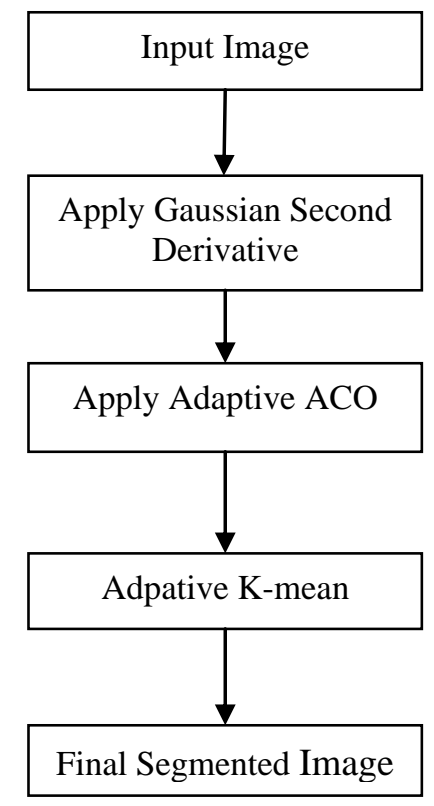

Fig1. Block Diagram of Proposed Model

\subsection{Proposed Algorithm}

1. Consider a gray image with any size as an N X N.

2. Find horizontal direction and vertical direction of an image can be obtained using the Gaussian second derivative method.

3. Initialize the base attractiveness, $\tau$, and visibility, gamma, $\eta$, for each edge.

4. For each iteration until its maximum value of fitness.

5. For each ant do this process.

6. Choose probability for ant then it moves to one location to another.

7. Store every location of ant in tabular list.

8. Repeat step 4 to 7 until each ant completed a solution.

9. For each ant find a best solution.

10. Update attractiveness for each edge that the ant moved

11. Repeat step 8 to 9 until traversal is completed.

12. If the local best solution is better than global solution, then save local best solution as a global solution.

13. Repeat Step 12 until best solution is found.

14. Repeat Step 4 to step 13 until finding the best location meets.

15. Select the number of desired clusters $k$. Arbitrarily place the $k$ cluster centers at different initial locations in the image. 
16. Assign each data point to the cluster whose center is closest.

17. Recompute the cluster centers; the cluster center should be at the average coordinates (center of gravity) of the data points that make up the cluster.

18. Go to step 16 until no more changes occur or a maximum number of iterations is reached.

Image Dataset

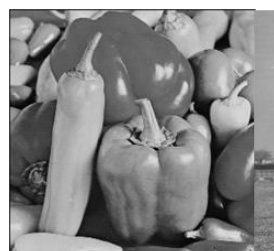

(A)

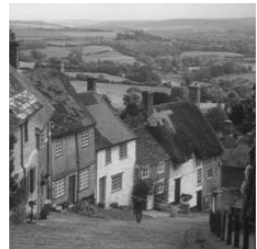

(D)

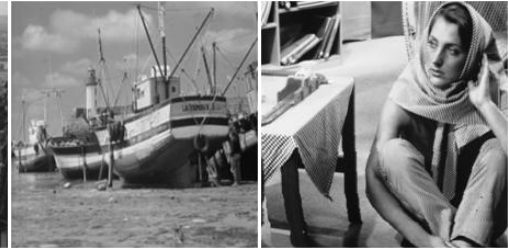

(E)

(F)
Fig2. Image Dataset

Performance Results of image (c)

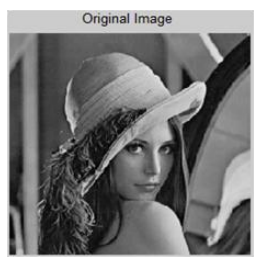

(a)

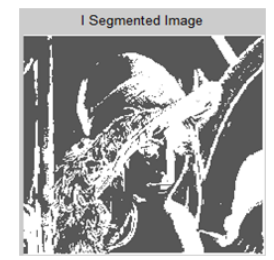

(c)

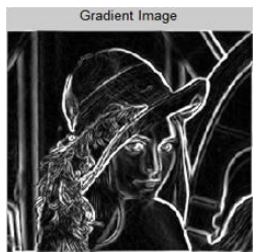

(b)

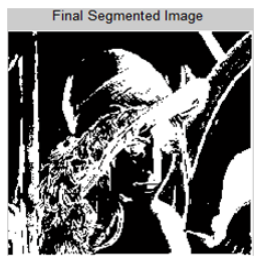

(d)
Fig3. (a) Original Image (b) Gradient Image (c) First Segmented Image using ACO (d) Second Segmented Image using our proposed method

Performance Results of image (a)

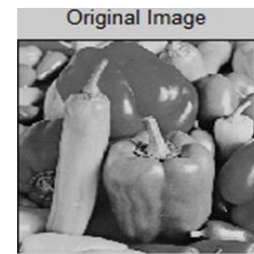

(a)

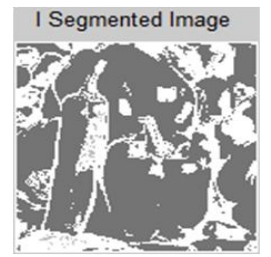

(c)

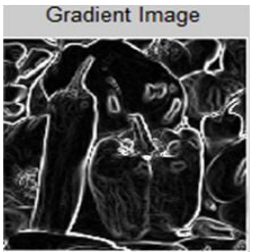

(b)

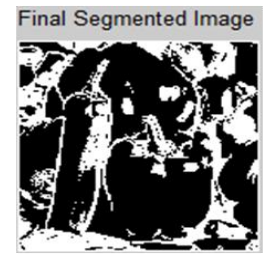

(d)
Fig4. (a) Original Image (b) Gradient ImagWe (c) First Segmented Image using ACO (d) Second Segmented Image using our proposed method

\section{RESULT ANALYSIS}

The experiment result was evaluated on MATLAB12a. In Table1, calculate the execution time for base and proposed system. The proposed time is better as compared to base algorithm. In this study, the execution time has reached up to 0.0275 seconds. In Table2, estimate the F - measure of accuracy performance of an image. This algorithm gives good accuracy in comparison to base method.

Table1. Execution Time Comparison between Base [4] and Proposed System

\begin{tabular}{|c|l|l|}
\hline $\begin{array}{c}\text { Image } \\
\text { Name }\end{array}$ & $\begin{array}{l}\text { Base } \\
\text { Time }\end{array}$ & $\begin{array}{l}\text { Proposed } \\
\text { Time }\end{array}$ \\
\hline (A) & 0.0910 & 0.0247 \\
\hline$(B)$ & 0.1050 & 0.0225 \\
\hline$(C)$ & 0.0916 & 0.0275 \\
\hline$(D)$ & 0.2108 & 0.1464 \\
\hline$(E)$ & 0.0924 & 0.0173 \\
\hline (F) & 0.2536 & 0.1889 \\
\hline
\end{tabular}

Table2. F-measure Comparison between Base[4] and Proposed System

\begin{tabular}{|c|c|c|}
\hline $\begin{array}{c}\text { Image } \\
\text { Name }\end{array}$ & $\begin{array}{c}\text { Base } \\
\text { F-measure }\end{array}$ & $\begin{array}{c}\text { Proposed } \\
\text { F-measure }\end{array}$ \\
\hline$(\mathrm{A})$ & 55.5119 & 68.6411 \\
\hline$(\mathrm{B})$ & 56.4330 & 69.7436 \\
\hline$(\mathrm{C})$ & 54.1312 & 66.9141 \\
\hline$(\mathrm{D})$ & 53.7792 & 65.5370 \\
\hline$(\mathrm{E})$ & 54.0146 & 71.6525 \\
\hline$(\mathrm{F})$ & 55.1361 & 72.9979 \\
\hline
\end{tabular}




\section{CONCLUSION}

The objective of segmentation is to make simpler and modify the demonstration of an image into something that is more important and easier to observe. In the image segmentation process, it is used to establish objects and background images. In this paper, work on techniques such as Ant Colony Optimization, Gaussian second derivative and adaptive Kmeans. Adaptive K-mean method can find the image segment faster than Fuzzy C-means. The proposed algorithm calculates the starting cluster $\mathrm{k}$ parameter to decrease the execution time. The experimental outcomes show that minimum time, and its quality has been demonstrated in several experiments.

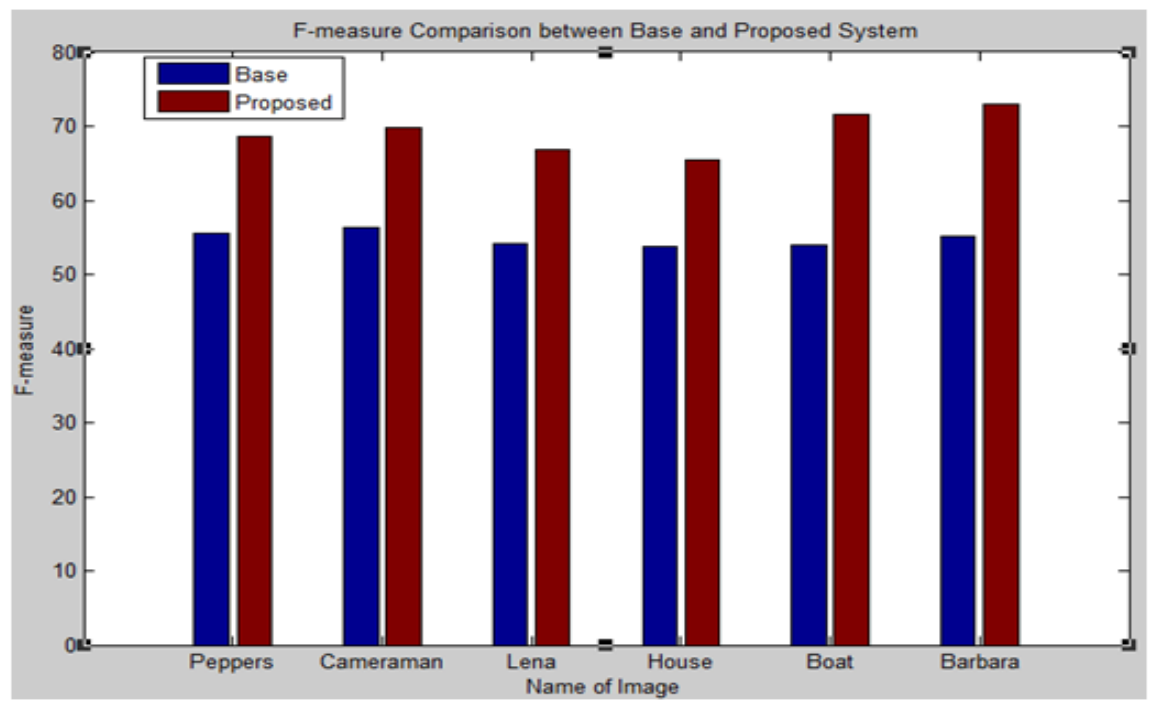

\section{Graph1. F-measure Comparison between Base[4] and Proposed System}

\section{REFERENCES}

[1] Ina Singh, Asst. Prof. Neelakshi Gupta ," Segmentation of Liver using Hybrid K-means Clustering and Level Set ",International Journal of Advanced Research in Computer Science and Software Engineering, Volume 5, Issue 8, August 2015, pp:742-746

[2] Varshali Jaiswal and Aruna Tiwari," A Survey of Image Segmentation based on Artificial Intelligence and Evolutionary Approach", IOSR Journal of Computer Engineering (IOSR-JCE), 8727Volume 15, Issue 3 (Nov. - Dec. 2013), PP 71-78

[3] Vrushali D. Mendhule , Gaurav Soni, Alesh Sharma," Interactive Image Segmentation Using Combined MRF and Ant Colony Optimization", International Journal Of Engineering And Computer Science ISSN:2319-7242 Volume 4 Issue 6 June 2015, Page No. 12281-12288

[4] Jue Lu and Rongqiang $\mathrm{Hu}, " \mathrm{~A}$ new hybrid clustering algorithm based on K-means and ant colony algorithm",Proceedings of the 2nd International Conference on Computer Science and Electronics Engineering (ICCSEE 2013), pp:1718-1721.

[5] Vahidsolemani, FarnoHeidariVincheh,“ Improving Ant Colony Optimization for Brain MRI Segmentation andBrain Tumor Diagnosis", IEEE 2013, 978-1-46736206-1/13.

[6] Ms. Aparna K and Dr. Mydhili K Nair,"A Detailed Study and Analysis of different Partitional Data Clustering
Techniques",International Journal of Innovative Research in Science, Engineering and Technology, Vol. 3, Issue 1, January 2014, pp: 8353-8359.

[7] Dharmendra K. Roy and Lokesh Sharma, " Genetic KMeans Clustering algorithm for mixed and categorical data sets", IJAIA 2010.

[8] N. Shi, "Research on K-Means Clustering Algorithms: An improved K-Means Algorithms", Intelligent Information Technology and Security Informatics, 63-67, IEEE 2010.

[9] Ina Singh, Asst. Prof. Neelakshi Gupta “ Segmentation of Liver using Hybrid K-means Clustering and Level Set" International Journal of Advanced Research in Computer Science and Software Engineering 5(8), August- 2015, pp. 742-746 @ 2015, IJARCSSE

[10] Bhagwati Charan Patel, Dr. G.R.Sinha "An Adaptive K-means Clustering Algorithm for Breast Image Segmentation" International Journal of Computer Applications (0975 - 8887) Volume 10- N.4, November 2010

[11] A. M. Khan, Ravi. S," Image Segmentation Methods: A Comparative Study", International Journal of Soft Computing and Engineering (IJSCE) ISSN: 2231-2307, Volume-3, Issue-4, September 2013, pp:84-92. 